\title{
Risk-benefit value of upper extremity function by an implanted electrical stimulation device targeting chronic cervical spinal cord injury
}

\author{
Kim D. Anderson ${ }^{1,2} \cdot$ Anne M. Bryden ${ }^{2,3} \cdot$ Megan Moynahan ${ }^{2}$ \\ Received: 28 May 2019 / Revised: 2 July 2019 / Accepted: 9 July 2019 \\ (c) The Author(s), under exclusive licence to International Spinal Cord Society 2019
}

\begin{abstract}
Study design A cross-sectional stated-preference survey using direct-assessment questions.

Objective To determine the relative value placed on different outcomes to be used in a pivotal trial for the upper extremity configuration of the Networked Neuroprosthesis (NNP) as well as the tolerance of the expected adverse event profile.

Setting Academic medical center in the United States.

Methods Distribution of an online survey to adults living with tetraplegia; extent of agreement with each question/statement was obtaining using a 1-7 Likert scale.

Results There were 8 statements about potential benefits in arm/hand function; for all statements, more than $70 \%$ of participants rated the functions as "1-very important" to regain. There were variable degrees of concern related to risks that could occur during the 30-day post-surgical period and increasing degrees of concern related to risks that could occur in the first 5 years, potentially due to the device, based on the increasing degree of invasiveness of the intervention required to address the event. When analysing the results based on all degrees of interest, more than $64 \%$ of responders were interested in getting the NNP with a success rate threshold as low as 50\% regardless of time post-injury. Chi-squared analyses revealed some associations between responses and sex, injury level, and injury duration; however, none of these were statistically significant upon post-hoc analysis.

Conclusion Data here indicate that people with tetraplegia are highly interested in a range of arm/hand functions and are tolerant of expected risks that may be associated with implanted neuroprosthetics.

Sponsorship The Institute for Functional Restoration funded this project through a sub-contract to K.D. Anderson from a larger Special Projects Award (grant number FP0020773) from the Craig H. Neilsen Foundation.
\end{abstract}

Supplementary information The online version of this article (https:// doi.org/10.1038/s41394-019-0213-9) contains supplementary material, which is available to authorized users.

$\triangle \operatorname{Kim}$ D. Anderson

kxa304@case.edu

1 Miami Project to Cure Paralysis, Department of Neurological Surgery, University of Miami School of Medicine, Miami, FL, USA

2 Institute for Functional Restoration, Case Western Reserve University, Cleveland, OH, USA

3 Department of Orthopaedics, MetroHealth Medical Center, Case Western Reserve University, Cleveland, OH, USA

\section{Introduction}

Electrical stimulation has been used for many decades to activate paralyzed muscles. Muscle activation can be achieved by placement of electrodes on the surface of the skin or implanted within the muscle, near the motor point. Implanted neural stimulation devices (neuroprosthetics) have been used in spinal cord injury (SCI) for over 30 years. One implanted neuroprosthesis targeting hand grasp in individuals with tetraplegia, the Freehand system, was approved by the FDA in 1997 (PMA \#P950035) and commercialized by NeuroControl Corporation $[1,2]$. The Freehand was demonstrated to be safe and effective, with users showing improvements in pinch force, ability to manipulate more every day objects, improved independence in activities of daily living, and positive user satisfaction [3]. 
Limitations of the system were the requirement of external components, and being restricted to eight stimulations channels, which only enabled two grasp patterns, palmar and lateral. The control method for user operation required movement of the opposite shoulder to control grasp as well as lock and unlock grasp patterns. To address the described limitations, second [4] and now third [5] generation systems have been created. The second generation system, the Case Western Reserve University Implanted Stimulator-Telemeter (IST), increased the number of stimulation channels to 10 (IST-10) and later, 12 (IST-12), permitting additional functions such as forearm pronation and elbow extension, as well as customized grasps. It also internalized the control source, allowing the user to control operation based on myoelectric feedback in the same arm [4]. The third generation system, the Case Western Reserve University Networked Neuroprosthesis (NNP) [5], has no externally worn components and features a modular design, allowing the distribution of stimulators and electrodes throughout the body, enabling multiple functions to be restored.

Our interest is in exploring the willingness of people with SCI to accept the risks and benefits of this third-generation system when configured to provide upper extremity (UE) function (e.g., hand grasp). The goal of this survey was to query a large number of people living with tetraplegia to characterize the relative risk:benefit value and inform the design of a future pivotal trial for the UE configuration of the NNP.

\section{Methods}

\section{Survey design}

A stated-preference study was performed utilizing directassessment questions. Stated-preference methodology refers to a family of techniques that use statements of individual respondents about their preferences to a set of hypothetical attributes [6-8]. The underlying theoretical framework of stated-preference methodology is ordinal-utility theory. Respondents are presented with a series of hypothetical choice problems and asked to state how they would behave in the situation. Ordinal-utility enables one to identify one preference over another, but cannot provide any information about the strength of preferences.

The survey questions were designed to explore perceptions of both risk and benefit of the NNP using estimates about its performance based on the earlier generation NeuroControl Freehand System, as reported in the product's approved labeling. Three categories of questions were included: (1) benefits of arm and hand function, (2) concern level of different adverse events that could potentially occur during the 30-day post-surgical period, and within the first 5 years after receiving the NNP implant, and (3) interest level in receiving a NNP given different threshold scenarios of benefit and risk. See Supplementary Material for exact phrasing of questions.

To explore perceptions of benefit, participants were presented with a range of probabilities $(90,80,65,50,10 \%)$ of achieving success and asked to rate their interest level in having the NNP implanted. The range of probabilities was chosen to span a realistic range of outcomes based on the Freehand study, plus lower probabilities in order to characterize worse case scenarios. Success was defined as achieving three specific outcomes with the neuroprosthetic: (1) an improvement in active movement of the arm, (2) an improvement in pinch or grasp strength in the hand, and (3) the ability to lift or move more everyday objects than before. This definition was selected because: (1) the NNP UE configuration has the potential to impact each function, (2) the clinical trial for the Freehand System used the same definition of success, and (3) this definition of success is being considered for a future pivotal clinical trial for the NNP UE configuration.

To explore perceptions of risk, we identified a low, medium, and high probability of experiencing types of risks associated with the acute (30 days) and long-term (5 years) use of the Freehand system. The medium point estimates were taken from the actual adverse event rate observed in the Freehand clinical trial. The low point estimate was then calculated by dividing the observed rate by two; and the high point estimate was established by doubling the observed rate. In two questions where the observed adverse event rate was already very low (1-2\%), the observed rate was used as the low point estimate, with the medium and high estimates derived by multiplying this by two and four respectively. See the Supplementary Material for details. Using a 1-7 Likert rating scale, participants were asked to identify the extent to which they agreed with or were concerned about the types and rates of adverse events. There were also seven basic demographic questions.

The questionnaire was administered online using the software program SurveyMonkey ${ }^{\mathrm{TM}}$ (San Mateo, CA, USA). A detailed description of SurveyMonkey ${ }^{\mathrm{TM}}$ security features is available at www.surveymonkey.com/mp/policy/ security/. All responses were immediately stored in a secured, computerized database. In an effort to not exclude individuals who did not have access to the Internet, the option of completing the survey via the postal mail or by telephone was provided; answers were immediately entered into the online survey upon receipt.

All survey questions and answer choices are available in the Supplementary Material. The study was reviewed and approved by the University of Miami Institutional Review Board, where the corresponding author was located at the 
time the survey was conducted. We certify that all applicable institutional and governmental regulations concerning the ethical use of human volunteers were followed during the course of this research.

\section{Participant recruitment}

To qualify for the study, individuals had to have a SCI, be 18 years of age or older, and have tetraplegia (via selfreport). A waiver of signed consent was granted by the institution. All of the elements of informed consent were described on the study information page, which was displayed on the first page of the survey. Consent and qualification were inferred by agreement and further participation in the survey. Those individuals who did not agree to the information provided on the study information page elected to not complete the survey. Participants were recruited by word of mouth through people living with SCI, postings on the internet, and individuals in the Miami Project volunteer registry. Enrollment occurred between August and November 2017.

\section{Data reporting and analyses}

All descriptive sample demographics were reported following the international SCI basic and core data set recommendations $[9,10]$. For analytic purposes, all question responses were collapsed into three categories (positive, neutral, negative). Pearson and Likelihood Chi-square tests [11] were used to identify associations between question responses and sex, injury level (C1-4 vs C5-8), or injury duration group (0-5 YPI vs 6-15 YPI vs $16+$ YPI) $(\alpha=0.05)$. For each significant association, the chi-square statistic of each cell was evaluated against a chi-square critical value corresponding to a Bonferonii corrected $\mathrm{p}$ value, which adjusted for the number of cells in the table (i.e., number of evaluations performed). The chi-square critical values for post-hoc evaluation were 7.037 for $2 \times 3$ tables (gender and injury level; $p=0.008(\alpha=0.05 / 6$ cells)) and 7.879 for $3 \times 3$ tables (injury duration, $p=0.005$ $(\alpha=0.05 / 9$ cells $)$ ). Cell chi-square values greater than or equal to these values were accepted as statistically significant. Data used to compute all contingency tables are presented in the Supplementary Material.

\section{Results}

\section{Demographics}

The survey was emailed to 1424 individuals living with tetraplegia due to traumatic or non-traumatic cause and age $\geq 18$ years; $3 \%$ of those emails bounced back, $4 \%$ opted
Table 1 Demographics

\begin{tabular}{|c|c|c|}
\hline \multirow[t]{2}{*}{ Sex } & Male & $78 \%(N=317)$ \\
\hline & Female & $22 \%(N=91)$ \\
\hline Age & $43 \pm 14$ years & \\
\hline \multirow[t]{7}{*}{ Cause of SCI } & Transport & $45 \%(N=185)$ \\
\hline & Sports & $30 \%(N=121)$ \\
\hline & Fall & $12 \%(N=50)$ \\
\hline & Other traumatic & $6 \%(N=23)$ \\
\hline & Assault & $3 \%(N=13)$ \\
\hline & Non-traumatic & $3 \%(N=11)$ \\
\hline & Unknown & $1 \%(N=5)$ \\
\hline \multirow[t]{2}{*}{ Location of SCI } & Cervical $1-4$ & $33 \%(N=135)$ \\
\hline & Cervical 5-8 & $67 \%(N=273)$ \\
\hline \multirow[t]{4}{*}{ Years post-Injury (YPI) } & $13 \pm 12$ years & \\
\hline & $0-5$ yrs & $32 \%(N=129)$ \\
\hline & $6-15$ yrs & $37 \%(N=151)$ \\
\hline & $16+y r s$ & $31 \%(N=128)$ \\
\hline \multirow[t]{6}{*}{ Primary mode of mobility } & Power wheelchair & $56 \%(N=230)$ \\
\hline & Scooter & $1 \%(N=5)$ \\
\hline & Manual w/c & $25 \%(N=104)$ \\
\hline & Power-assist w/c & $6 \%(N=23)$ \\
\hline & Walk w/ devices & $6 \%(N=24)$ \\
\hline & Walk independent & $5 \%(N=22)$ \\
\hline \multirow[t]{7}{*}{ Secondary mode of mobility } & No & $51 \%(N=210)$ \\
\hline & Power wheelchair & $11 \%(N=44)$ \\
\hline & Scooter & $1 \%(N=5)$ \\
\hline & Manual w/c & $22 \%(N=89)$ \\
\hline & Power-assist w/c & $6 \%(N=24)$ \\
\hline & Walk w/ devices & $7 \%(N=29)$ \\
\hline & Walk independent & $2 \%(N=7)$ \\
\hline
\end{tabular}

out, and $32 \%$ responded $(N=457)$. Of the responders, 49 did not provide answers to all of the questions so the analyses presented here are for the 408 participants who completed the entire survey. The population was $78 \%$ male with $67 \%$ reporting cervical 5-8 lesion level. The mean age and standard deviation of the population was $43 \pm 14$ years (43, 18-83 [median, range]) and the mean post-injury duration was $13 \pm 12$ years $(9,<1-82$ [median, range]). The primary cause of injury, distribution of post-injury duration, primary mode of mobility, and secondary mode of mobility are provided in Table 1.

\section{Functional improvement}

There were 8 statements about improved UE function; for all statements, more than $70 \%$ of participants rated the functions as "1-very important" to regain (Fig. 1). There was no statistical difference in association between sex and question responses (Table 2). There were limited associations between injury level or injury duration and responses 
Fig. 1 Importance of upper extremity functions. (ADLs activities of daily living, $w / o$ without)

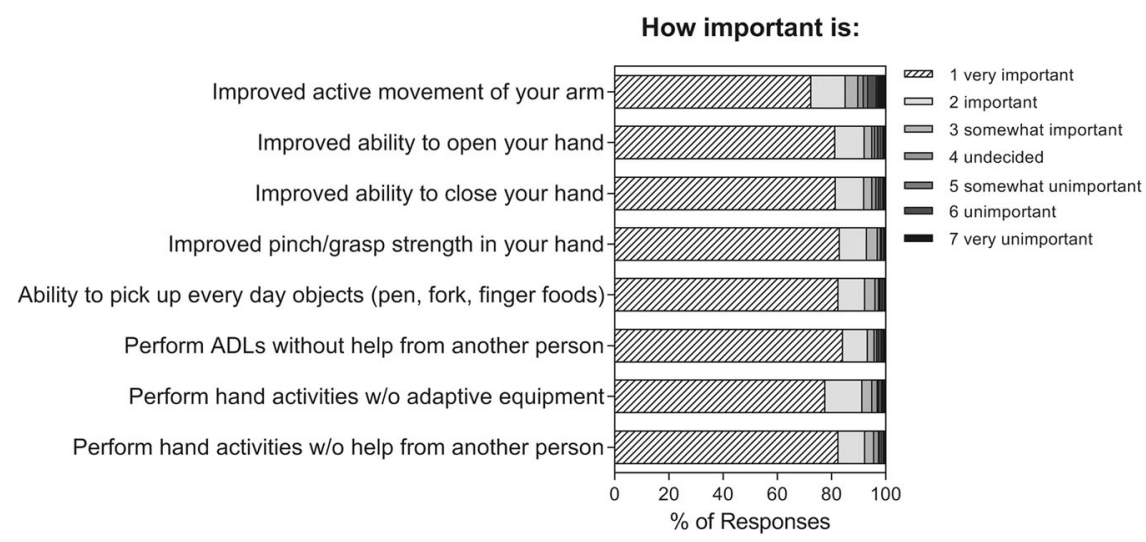

(Table 2), but post-hoc analyses did not reveal where those differences were located. Visual inspection of the data did not reveal any trends between groups (see Supplementary Material).

\section{Potential risks}

The next topic of inquiry was about possible risks that could be associated with implanted devices such as the NNP. There were variable degrees of concern related to risks that could occur during the 30-day post-surgical period (Fig. 2a). There was no difference in association between injury duration and question responses (Table 2). There were limited associations between sex or injury level and responses (Table 2), but post-hoc analyses did not reveal where those differences were located. Visual inspection of the data suggested a trend for females and people with C5-8 injury levels to be more conservative about risk than men or people with C1-4 injury levels (see Supplementary Material). The second category of risks included those that could occur during the first 5 years and potentially be related to the implant. There were increasing degrees of concern related to these risks based on the increasing degree of invasiveness of the intervention required to address the event (Fig. 2b). There was no difference in association between sex, injury level, or injury duration and question responses (Table 2).

\section{Threshold scenarios}

The first threshold scenario was designed to investigate the tolerability of different thresholds of success. Figure 3 shows the results for all respondents. There was no difference in association between injury level and question responses (Table 2). There were multiple associations between sex and responses and two associations between injury duration and responses (Table 2), but post-hoc analyses did not reveal where those differences were located. Visual inspection of the data suggested a trend for females and people who were 16 or more years' post-injury to be more conservative about risk than men or people with shorter injury durations (see Supplementary Material).

The second threshold scenario was designed to investigate the tolerability of different thresholds of risk. For all respondents, Fig. 4 shows the threshold variance related to the short-term post-surgical risks and Fig. 5 shows the threshold variance for the later device-related risks. Interest levels increased or decreased as risk probabilities decreased or increased. There was only 1 difference in association between injury level and question responses (Table 2). There were multiple associations between sex and responses and several associations between injury duration and responses (Table 2), but post-hoc analyses did not reveal where those differences were located. Visual inspection of the data suggested a consistent trend for females and people who were 16 or more years' post-injury to be more conservative about risk than men or people with shorter injury durations (see Supplementary Material).

\section{Discussion}

These results indicate that individuals living with tetraplegia are very interested in interventions that target UE function. Further, they are tolerant to varying degrees of potential risks associated with implanted neuroprosthetic devices. There were trends for differences in risk tolerance based on sex and time post-injury, but when put in the context of the estimated probability of experiencing a particular risk, there was overall interest in receiving an implanted neuroprosthetic device that can provide similar outcomes as the Freehand system, for both benefits and risks.

\section{Functional improvement}

We asked participants about eight improvements in the UE that the NNP could potentially provide. All functions were rated overwhelmingly as very important, almost to the point 
Table 2 Chi-squared analyses of associations between question responses and sex, injury level, and injury duration

\begin{tabular}{|c|c|c|c|c|}
\hline Category & Description & $\operatorname{Sex}^{\mathrm{a}}$ & Injury level $^{\mathrm{a}}$ & Injury duration $^{\mathrm{b}}$ \\
\hline \multirow[t]{8}{*}{ Benefits } & Improved active movement of arm & NS & NS & NS \\
\hline & Open your hand & NS & $\mathrm{L} \chi^{2} 10.49, p=0.005$ & NS \\
\hline & Close your hand & NS & $\mathrm{L} \chi^{2} 9.04, p=0.011$ & $\mathrm{~L} \chi^{2} 12.64, p=0.013$ \\
\hline & Pinch or grasp strength in your hand & NS & NS & NS \\
\hline & Pick up everyday objects & NS & NS & NS \\
\hline & ADLs w/o help from person & NS & NS & NS \\
\hline & Hand activities w/o adaptive equipment & NS & NS & NS \\
\hline & Hand activities w/o person or equipment & NS & NS & NS \\
\hline \multirow[t]{9}{*}{ Risks } & Post-surgical infection that requires treatment & $\mathrm{P} \chi^{2} 10.22, p=0.006$ & NS & NS \\
\hline & Temporary, post-surgical pain & NS & NS & NS \\
\hline & Temporary reduction in arm or hand function & NS & $\mathrm{P} \chi^{2} 6.93, p=0.031$ & NS \\
\hline & Corrected without surgery & NS & NS & NS \\
\hline & Outpatient surgery & NS & NS & NS \\
\hline & Inpatient surgery & NS & NS & NS \\
\hline & Remove 1 or more components & NS & NS & NS \\
\hline & Remove entire system & NS & NS & NS \\
\hline & Replace battery every 5 year & NS & NS & NS \\
\hline \multicolumn{5}{|c|}{ Thresholds } \\
\hline \multirow[t]{5}{*}{ Success } & Interest in NNP if $90 \%$ chance of achieving success & NS & NS & $\mathrm{L} \chi^{2} 10.26, p=0.036$ \\
\hline & Interest in NNP if $80 \%$ chance of achieving success & $\mathrm{L} \chi^{2} 7.69, p=0.021$ & NS & NS \\
\hline & Interest in NNP if $65 \%$ chance of achieving success & $\mathrm{P} \chi^{2} 5.56, p=0.062$ & NS & $\mathrm{P} \chi^{2} 10.71, p=0.030$ \\
\hline & Interest in NNP if 50\% chance of achieving success & $\mathrm{P} \chi^{2} 7.02, p=0.029$ & NS & NS \\
\hline & Interest in NNP if $10 \%$ chance of achieving success & $\mathrm{P} \chi^{2} 11.39, p=0.003$ & NS & NS \\
\hline \multirow[t]{25}{*}{ Risk } & Interest in NNP if $1 \%$ chance of infection & NS & NS & $\mathrm{L} \chi^{2} 14.45, p=0.006$ \\
\hline & Interest in NNP if $2 \%$ chance of infection & $\mathrm{P} \chi^{2} 7.51, p=0.023$ & NS & $\mathrm{P} \chi^{2} 12.07, p=0.017$ \\
\hline & Interest in NNP if $4 \%$ chance of infection & $\mathrm{P} \chi^{2} 9.50, p=0.083$ & NS & $\mathrm{P} \chi^{2} 10.68, p=0.030$ \\
\hline & Interest in NNP if $10 \%$ chance of pain & NS & NS & $\mathrm{L} \chi^{2} 12.97, p=0.011$ \\
\hline & Interest in NNP if $20 \%$ chance of pain & NS & NS & $\mathrm{P} \chi^{2} 10.19, p=0.037$ \\
\hline & Interest in NNP if $40 \%$ chance of pain & NS & NS & $\mathrm{P} \chi^{2} 16.63, p=0.002$ \\
\hline & Interest in NNP if temp reduction arm function & NS & NS & $\mathrm{P} \chi^{2} 10.58, p=0.032$ \\
\hline & Interest in NNP if $25 \%$ chance fix w/o surgery & NS & NS & NS \\
\hline & Interest in NNP if $50 \%$ chance fix w/o surgery & NS & NS & NS \\
\hline & Interest in NNP if $100 \%$ chance fix w/o surgery & NS & NS & NS \\
\hline & Interest in NNP if $5 \%$ chance fix w/ Outpt surgery & NS & NS & NS \\
\hline & Interest in NNP if $10 \%$ chance fix w/ Outpt surgery & $\mathrm{P} \chi^{2} 8.15, p=0.017$ & NS & NS \\
\hline & Interest in NNP if $20 \%$ chance fix w/ Outpt surgery & NS & NS & NS \\
\hline & Interest in NNP if $10 \%$ chance fix w/ Inpt surgery & $\mathrm{P} \chi^{2} 9.23, p=0.009$ & NS & NS \\
\hline & Interest in NNP if $20 \%$ chance fix w/ Inpt surgery & NS & NS & $\mathrm{P} \chi^{2} 10.83, p=0.029$ \\
\hline & Interest in NNP if $40 \%$ chance fix w/ Inpt surgery & NS & NS & $\mathrm{P} \chi^{2} 10.18, p=0.038$ \\
\hline & Interest in NNP if $5 \%$ chance remove $1+$ parts & $\mathrm{P} \chi^{2} 6.89, p=0.032$ & NS & NS \\
\hline & Interest in NNP if $10 \%$ chance remove $1+$ parts & NS & NS & $\mathrm{P} \chi^{2} 13.64, p=0.009$ \\
\hline & Interest in NNP if $20 \%$ chance remove $1+$ parts & NS & NS & $\mathrm{P} \chi^{2} 11.56, p=0.021$ \\
\hline & Interest in NNP if $2 \%$ chance remove All parts & $\mathrm{P} \chi^{2} 7.52, p=0.023$ & NS & $\mathrm{P} \chi^{2} 10.64, p=0.031$ \\
\hline & Interest in NNP if $4 \%$ chance remove All parts & NS & NS & NS \\
\hline & Interest in NNP if $8 \%$ chance remove All parts & $\mathrm{P} \chi^{2} 12.30, p=0.002$ & NS & NS \\
\hline & Interest in NNP if replace battery $3 \mathrm{yr}$ w/ Outpt surgery & $\mathrm{P} \chi^{2} 7.19, p=0.027$ & NS & $\mathrm{P} \chi^{2} 12.16, p=0.016$ \\
\hline & Interest in NNP if replace battery $5 \mathrm{yr}$ w/ Outpt surgery & NS & NS & $\mathrm{P} \chi^{2} 11.41, p=0.022$ \\
\hline & Interest in NNP if replace battery $7 \mathrm{yr}$ w/ Outpt surgery & $\mathrm{P} \chi^{2} 6.69, p=0.035$ & $\mathrm{P} \chi^{2} 9.98, p=0.007$ & NS \\
\hline
\end{tabular}

adegrees of freedom $=2$

${ }^{\mathrm{b}}$ degrees of freedom $=4$

$\chi$ and $p$ values are not corrected for multiple comparisons

$N S$ not significant, $P \chi^{2}$ Pearson's chi-square, $L \chi^{2}$ Likelihood ratio chi-square, Outpt outpatient, Inpt inpatient, $N N P$ networked Neuroprosthesis 
Fig. 2 Concern about risks potentially associated with implanted devices like the Networked Neuroprosthesis (NNP). a Degree of concern about risks that could occur within the 30-day post-surgical period and be associated with the surgical procedure or location of implant. b Degree of concern about risks that could occur during the first 5 years and potentially be related to the implant. ( $w / o$ without, $w /$ with, surg surgery, outpt outpatient, inpt inpatient, $1+=1$ or more)
A

How concerned would you be about:

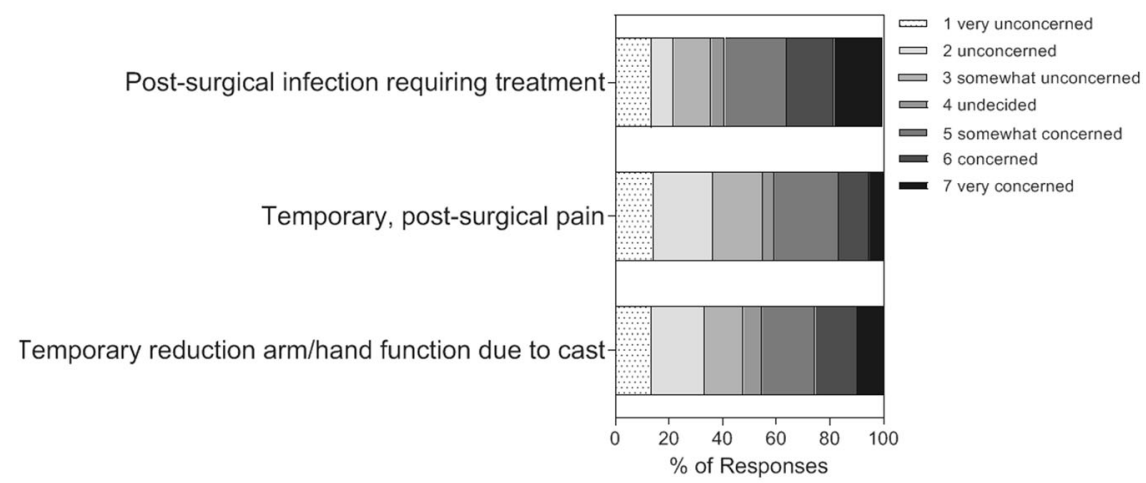

B

How concerned would you be about:

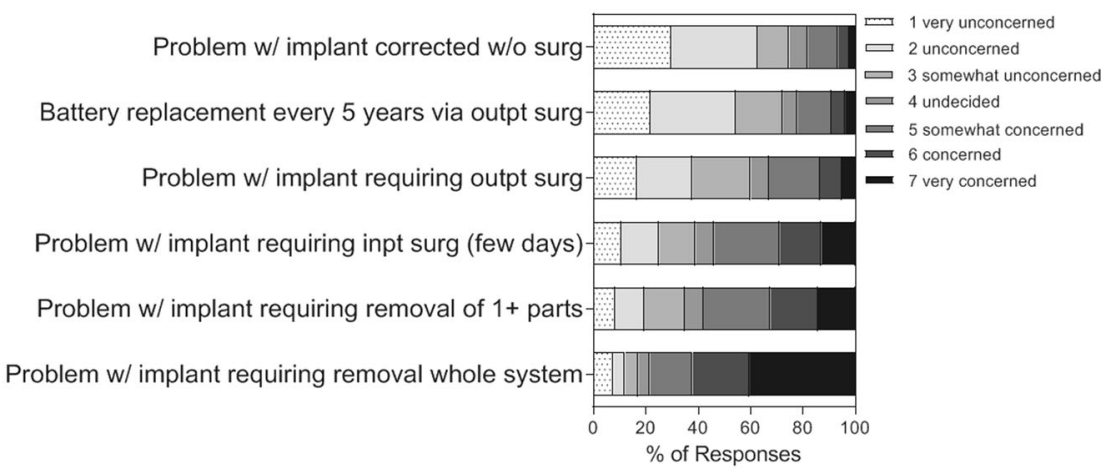

Knowing the definition of success, how interested would you be in getting NNP if...

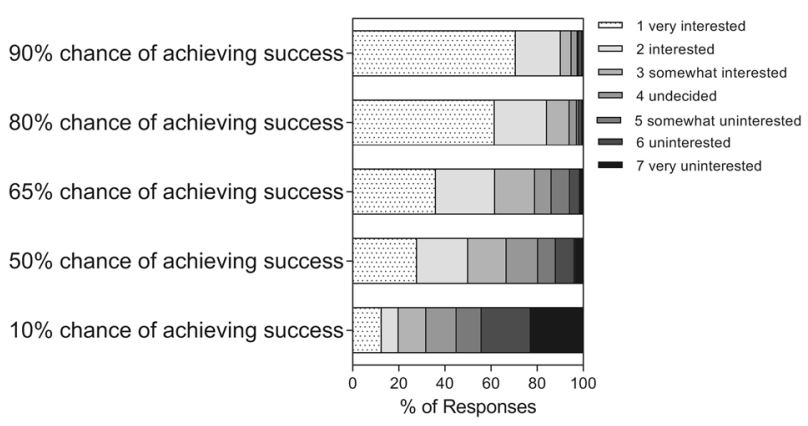

Fig. 3 Participant interest in the Networked Neuroprosthesis (NNP) in different scenarios of success

of appearing as a ceiling effect. It is unlikely that these responses are strictly related to the NNP device. We postulate that any intervention that could impact those functions would likely also be rated as highly important by people with tetraplegia. Examples of such interventions could include nerve transfers, tendon transfers, surface FES, implanted neuroprosthetics, potential drugs, and biologics targeting UE function.

Regaining UE function has been documented as a high priority for people with tetraplegia for many years. Anderson [12] prospectively queried 681 people living with chronic
SCI to identify functional return that would most improve quality of life. Fifty-one percent of respondents had tetraplegia and regaining arm/hand function was the highest ranked desired return (48.7\%). Donnelly [13] demonstrated retrospectively that in the early rehabilitation period self-care goals were most frequently cited as important by people with SCI. Snoek [14] performed a prospective survey in the Dutch and United Kingdom tetraplegic communities and determined that regaining hand function was very important to improving quality of life. A systematic review of perceived importance of life domains and priorities of people with SCI reinforced the finding that regaining arm and hand function was consistently a high priority for people living with tetraplegia [15]. This reinforces how important these functions are for people living with tetraplegia.

For each of the eight functions queried, there was also a small portion of responders that rated them as "somewhat unimportant", "unimportant", or "very unimportant". It is possible that those individuals already had adequate function in those activities and therefore rated them as unimportant to restore. This could be a reflection of the heterogeneity of cervical injuries, however the overall number of responders stating the UE functions were unimportant was small. These responses may also reflect a small proportion of responders not interested in any neurotechnology. 
A

How interested would you be in getting NNP if the risk of post-surgical infection requiring treatment was...

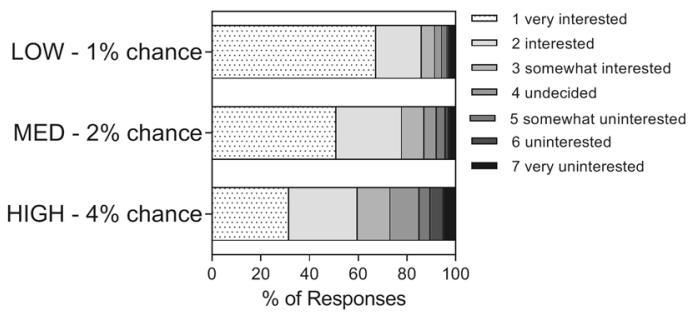

B

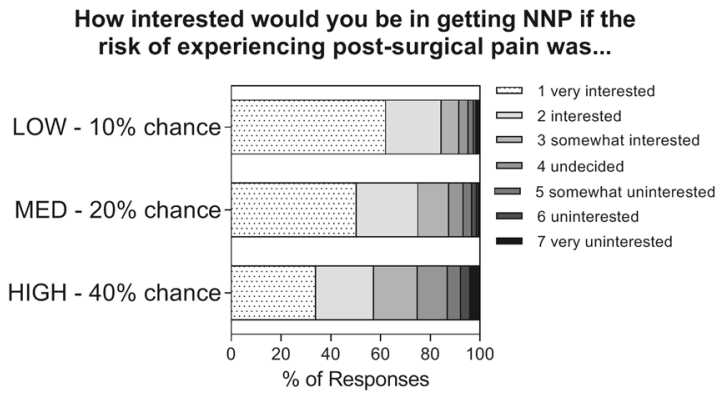

C How interested would you be in getting NNP if you experienced...

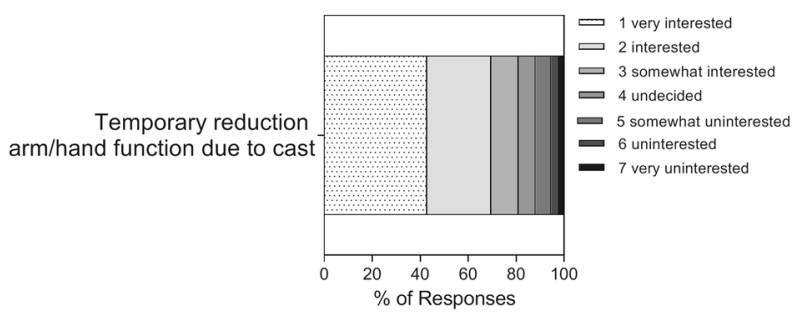

Fig. 4 Tolerability of risk thresholds during the first 30 days. a Interest in the Networked Neuroprosthesis (NNP) under low, medium, and high probability of experiencing a post-surgical infection requiring treatment. b Interest in the NNP under low, medium, and high probability of experiencing post-surgical pain. $\mathbf{c}$ Interest in the NNP if there were a temporary reduction in upper extremity function due to immobilization

\section{Risks and benefits}

When trading off the potential risks and benefits of the NNP, respondents were interested in receiving the system when the hypothetical outcomes matched the observed outcomes for the previously-FDA-approved NeuroControl Freehand system. However, even when the hypothetical outcome posed substantially more risk (e.g. doubling the adverse event rate over what was observed with Freehand), respondents still remained highly interested in receiving the system. This suggests that there is a high degree of risk tolerance for implantable neuroprosthetics that provide meaningful functional UE outcomes. It also suggests that an UE neuroprosthesis could enjoy widespread acceptance by the user community, within a fairly wide range of outcomes for a clinical trial.
Across several threshold scenarios, females and people injured 16 or more years were more conservative in their definition of success and risk tolerance. We postulate that this could be related to many complex factors worthy of further investigation. With increasing time post-injury, people develop a longer health experience related to their SCI. It is possible that they may be warier of interventions that could have a potential negative impact on their health. Similarly, they may be more likely to have an established routine that provides stability for them and may be hesitant to introduce change to that routine. It is also possible that they may have been compensating for many years to achieve degrees of function by whatever means possible and they may be fearful of losing those gains. These concepts were identified in a qualitative study evaluating readiness for stem cell clinical trials, and people with chronic SCI were less receptive to participating in such trials than people with subacute injuries for similar reasons as postulated above [16].

Further, people who have lived with their SCI for shorter durations may still be seeking improvements or interventions on a more active level and, therefore, may be more risk tolerant. Disease duration has been shown to impact risk tolerance in multiple sclerosis. Individuals with a longer disease duration demonstrate a greater tolerance of risk, presumably related to the progressive nature of multiple sclerosis $[17,18]$. SCI is not progressive, but just the opposite. Initially, this catastrophic event impacts every aspect of life and it is only with increasing time post-injury that people cope, adapt, and develop stability [19, 20]. Hence, their risk tolerance may mirror that process. Similarly, females living with multiple sclerosis have lower risk tolerance to therapies than males [18]. Further investigation related to reasons why females with SCI may have lower risk tolerance to therapies is required.

\section{Study limitations}

We acknowledge the limitations of this survey; it was administered primarily on-line. Our sample could therefore be biased towards persons who frequent the Internet. The Internet has become a prominent means of gathering resource information and has removed multiple barriers for many people living with disabilities worldwide [21, 22]. Previous studies show that people living with SCI prefer to receive research information from the Internet [23].

We did purposefully keep the survey short in an effort to make it less cumbersome and burdensome to participants. The primary limitation of stated-preference data is that the choices are hypothetical. Respondents may not know how they would actually respond when faced with the real situation of deciding to undergo implantation of the NNP, or any invasive procedure for that matter. Additionally, people responded to each attribute individually, not to a 
A How interested would you be in getting NNP if the risk of a problem $\mathrm{w} /$ implant that could be corrected w/o surgery was...

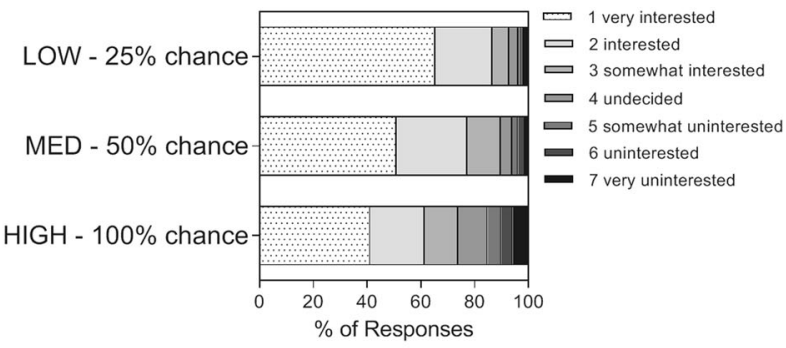

B How interested would you be in getting NNP if the risk of a problem $\mathrm{w} /$ implant that could be corrected $\mathrm{w} /$ outpatient surgery was...

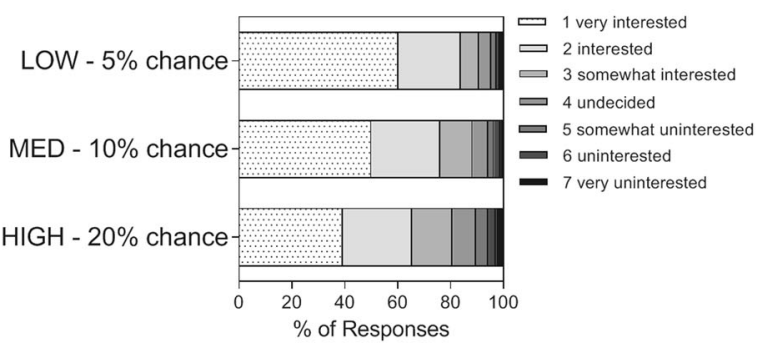

C How interested would you be in getting NNP if the risk of a problem $w /$ implant that could be corrected $w /$ inpatient surgery was...

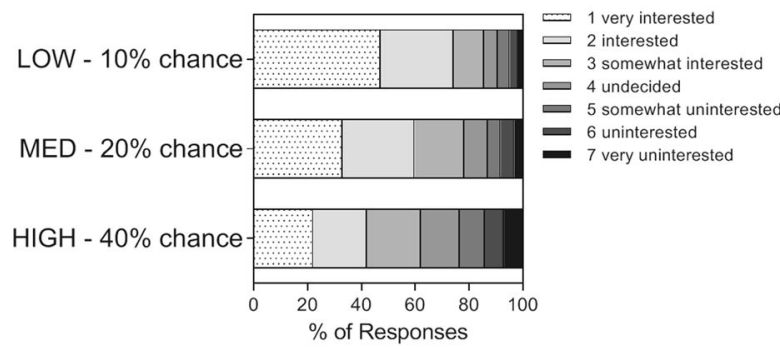

Fig. 5 Tolerability of risk thresholds during the first 5 years. Interest in the Networked Neuroprosthesis (NNP) under low, medium, and high probability of experiencing a problem a that could be corrected without surgery, b that could be corrected with outpatient surgery, c

profile of attributes. Future studies will involve methodology to gain information about tradeoffs people are willing to take related to various risk:benefit combinations.

\section{Conclusion}

Potential end users of investigational interventions not only will gain the benefits of such therapeutics, but also will bear any potential harms. Understanding how they weigh benefits and risks can help prioritize engineering decisions on design, device improvements, troubleshooting, clinical trial design,
D How interested would you be in getting NNP if the risk of a problem requiring removal of 1 or more components was...

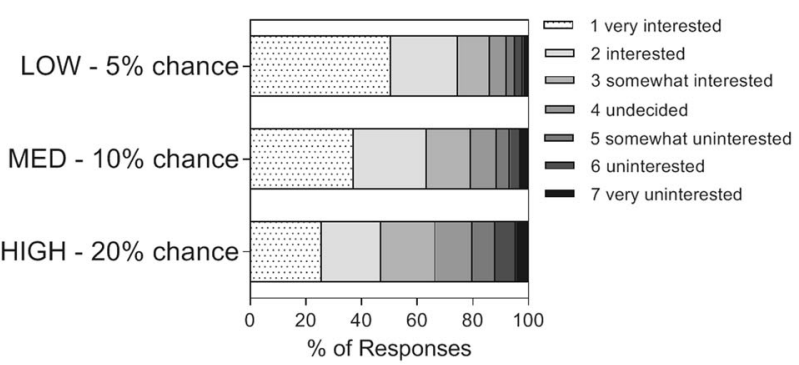

E How interested would you be in getting NNP if the risk of a problem requiring removal of the whole system was...

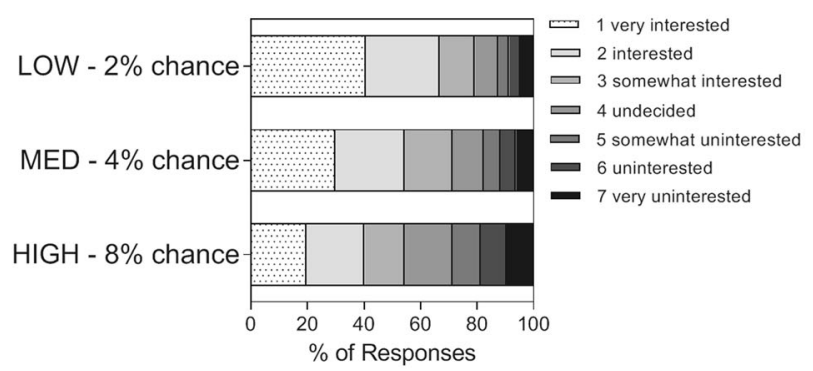

F How interested would you be in getting NNP if you had to replace the battery via outpatient surgery...

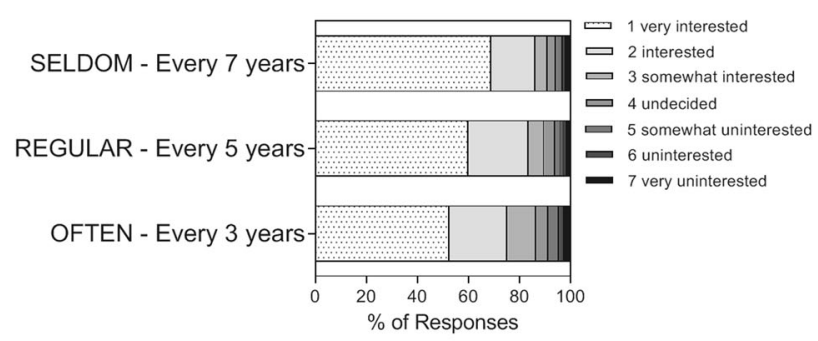

that could be corrected with inpatient surgery, $\mathbf{d}$ requiring removal of one or more components of the device, e requiring the removal of the whole system. f Interest in the NNP if the need to undergo outpatient surgery to replace the battery were every 3,5 , or 7 years

and functional targets. Therefore, it is critical to engage end users early and often in research and development of therapeutic interventions. Data here indicate that people with tetraplegia are highly interested in a range of UE benefits and are appropriately tolerant of expected risks associated with neuroprosthetics, but sex and injury duration may influence risk:benefit preferences.

\section{Data archiving}

All survey questions and answer options as well as the raw data used for all contingency tables are included in the 
Supplementary Material. A copy of the full dataset can be obtained by contacting the corresponding author.

Acknowledgements The authors would like to thank all the individuals living with SCI who took the time to provide input to this study. A special thanks is extended to Dr. Rachel Cowan for statistical advice. An abstract of preliminary analysis of this work has been published in the program book of the 2018 Tetrahand World Congress.

Funding The Institute for Functional Restoration funded this project through a sub-contract to KDA from a larger Special Projects Award (grant number FP0020773) from the Craig H. Neilsen Foundation.

\section{Compliance with ethical standards}

Conflict of interest The authors declare that they have no conflict of interest.

Publisher's note: Springer Nature remains neutral with regard to jurisdictional claims in published maps and institutional affiliations.

\section{References}

1. Kilgore KL, Peckham PH, Keith MW, Thrope GB, Wuolle KS, Bryden AM, et al. An implanted upper-extremity neuroprosthesis. Follow-up of five patients. J Bone Jt Surg Am. 1997;79:533-41.

2. Peckham PH, Keith MW, Kilgore KL, Grill JH, Wuolle KS, Thrope GB, et al. Efficacy of an implanted neuroprosthesis for restoring hand grasp in tetraplegia: a multicenter study. Arch Phys Med Rehabil. 2001;82:1380-8.

3. Wuolle KS, Van Doren CL, Bryden AM, Peckham PH, Keith M, Kilgore KL, et al. Satisfaction with and usage of a hand neuroprosthesis. Arch Phys Med Rehabil. 1999;80:206-13.

4. Kilgore KL, Hoyen HA, Bryden AM, Hart RL, Keith MW, Peckham PH. An implanted upper-extremity neuroprosthesis using myoelectric control. J Hand Surg Am. 2008;33:539-50.

5. Peckham PH, Kilgore KL. Challenges and opportunities in restoring function after paralysis. IEEE Trans Biomed Eng. 2013;60:602-9.

6. Lancaster KJ. A new approach to consumer theory. J Political Econ. 1966;74:132-47.

7. Kroes EP, Sheldon RJ. Stated preference methods. An Introduction. J Transp Econ Policy. 1988;22:11-25.

8. Ho M, Saha A, McCleary KK, Levitan B, Christopher S, Zandlo $\mathrm{K}$, et al. A framework for incorporating patient preferences regarding benefits and risks into regulatory assessment of medical technologies. Value Health. 2016;19:746-50.
9. DeVivo MJ, Biering-Sørensen F, New P, Chen Y. Standardization of data analysis and reporting of results from the International Spinal Cord Injury Core Data Set. Spinal Cord. 2011;45:596-9.

10. DeVivo M, Biering-Sørensen F, Charlifue S, Noonan V, Post M, Stripling $\mathrm{T}$, et al. International spinal cord injury core data set. Spinal Cord. 2006;44:535-40.

11. McHugh ML. The chi-square test of independence. Chi Sq Test Indep. 2013;23:143-9.

12. Anderson KD. Targeting recovery: Priorities of the spinal cord injured population. J Neurotrauma. 2004;21:1371-83.

13. Donnelly C, Eng JJ, Hall J, Alford L, Giachino R, Norton K, et al. Client-centred assessment and the identification of meaningful treatment goals for individuals with a spinal cord injury. Spinal Cord. 2004;42:302-7.

14. Snoek GJ, IJzerman MJ, Hermens HJ, Maxwell D, BieringSorensen F. Survey of the needs of patients with spinal cord injury: Impact and priority for improvement in hand function in tetraplegics. Spinal Cord. 2004;42:526-32.

15. Simpson LA, Eng JJ, Hsieh JTC, Wolfe DL. SCIRE Research Team. The health and life priorities of individuals with spinal cord injury: A systematic review. J Neurotrauma. 2012;29:1548-55.

16. Illes J, Reimer JC, Kwon BK. Stem cell clinical trials for spinal cord injury: Readiness, reluctance, redefinition. Stem Cell Rev. 2011;7:997-1005.

17. Glanz BI, Greeke E, LaRussa A, Stuart F, Rintell DJ, Chitnis T, et al. Risk attitudes and risk perceptions in individuals with multiple sclerosis. Mult Scler J Exp Transl Clin. 2016;2:2055217316665406.

18. Fox RJ, Salter A, Alster JM, Dawson NV, Kattan MW, Miller D, et al. Risk tolerance to MS therapies: survey results from the NARCOMS registry. Mult Scler Relat Disord. 2015;4:241-9.

19. Schwartz CE, Stucky B, Rivers CS, Noonan VK, Finkelstein JA. RHSCIR Network. Quality of life and adaptation in people with spinal cord injury: Response shift effects from 1 to 5 years postinjury. Arch Phys Med Rehabil. 2018;S0003-9993:30117-5.

20. Dibb B, Ellis-Hill C, Donovan-Hall M, Burridge J, Rushton D. Exploring positive adjustment in people with spinal cord injury. J Health Psychol. 2014;19:1043-54.

21. Rhodes SD, Bowie DA, Hergenrather KC. Collecting behavioural data using the world wide web: considerations for researchers. J Epidemiol Community Health. 2003;57:68-75.

22. Mitchell S, Ciemnecki A, CyBulski K, Markesich J, (eds). Removing barriers to survey participation for persons with disabilities. Ithaca, NY: Cornell University Rehabilitation Research and Training Center on Disability Demographics and Statistics; 2006.

23. Edwards L, Krassioukov A, Fehlings M. Importance of access to research information among individuals with spinal cord injury: results of an evidence-based questionnaire. Spinal Cord. 2002;40:529-35. 